Article

\title{
Perilla frutescens Leaf Alters the Rumen Microbial Community of Lactating Dairy Cows
}

\author{
Zhiqiang Sun, Zhu Yu and Bing Wang * (D) \\ College of Grass Science and Technology, China Agricultural University, Beijing 100193, China; \\ szq141835@sina.com (Z.S.); yuzhu33150@sina.com (Z.Y.) \\ * Correspondence: wangb@cau.edu.cn
}

Received: 25 September 2019; Accepted: 12 November 2019; Published: 13 November 2019

\begin{abstract}
Perilla frutescens (L.) Britt., an annual herbaceous plant, has antibacterial, anti-inflammation, and antioxidant properties. To understand the effects of $P$. frutescens leaf on the ruminal microbial ecology of cattle, Illumina MiSeq 16S rRNA sequencing technology was used. Fourteen cows were used in a randomized complete block design trial. Two diets were fed to these cattle: a control diet (CON); and CON supplemented with $300 \mathrm{~g} / \mathrm{d}$ P. frutescens leaf (PFL) per cow. Ruminal fluid was sampled at the end of the experiment for microbial DNA extraction. Overall, our findings revealed that supplementation with PFL could increase ruminal fluid $\mathrm{pH}$ value. The ruminal bacterial community of cattle was dominated by Bacteroidetes, Firmicutes, and Proteobacteria. The addition of PFL had a positive effect on Firmicutes, Actinobacteria, and Spirochaetes, but had no effect on Bacteroidetes and Proteobacteria compared with the CON. The supplementation with PFL significantly increased the abundance of Marvinbryantia, Acetitomaculum, Ruminococcus gauvreauii, Eubacterium coprostanoligenes, Selenomonas_1, Pseudoscardovia, norank_f_Muribaculaceae, and Sharpea, and decreased the abundance of Treponema_2 compared to CON. Eubacterium coprostanoligenes, and norank_f_Muribaculaceae were positively correlated with ruminal $\mathrm{pH}$ value. It was found that norank_f_Muribaculaceae and Acetitomaculum were positively correlated with milk yield, indicating that these different genera are PFL associated bacteria. This study suggests that PFL supplementation could increase the ruminal $\mathrm{pH}$ value and induce shifts in the ruminal bacterial composition.
\end{abstract}

Keywords: $16 \mathrm{~S}$ rRNA gene; cow; Perilla frutescens; rumen microbiota

\section{Introduction}

Plant secondary metabolites are naturally produced by plants or special medicinal herbs and can be readily formulated into feed rations [1]. The bioactive compounds derived from medicinal herbs have been discussed as potential alternatives to conventional antibiotics in ruminant production [2]. Perilla frutescens leaf (PFL) is a commonly used medicinal herb with antibacterial, antiallergy, anti-inflammatory, antioxidant, antidepressant, and anticancer properties due to its secondary metabolites including phenolic compounds, flavonoids, triterpenes, anthocyanin, and $\alpha$-linolenic acid $[3,4]$. The use of medicinal herbs and plant secondary metabolites in livestock has been a hot topic in veterinary research and practice [5], regarding the increasing concerns and challenges of using antimicrobials in livestock production and the proliferation of resistant bacteria via the food chain [6].

It has been established that the rumen microbial community has a profound impact on the performance, health, and immunity of the host animal [7,8]. Previous studies found that the effect of ruminal microbiota on the host can be achieved by the short chain fatty acids released by ruminal bacteria such as Mitsokella spp [9]. In addition, Shen et al. [10] found that the effect of rumen-derived short chain fatty acids on the growth and metabolism of epithelial cells was mediated by the regulatory network of G protein coupled receptor (GPR) and histone deacetylase (HDAC). Thus, the rumen 
microbiome has intimate connections with growth and development of the host animal. It has been found that the supplement of Andrographis paniculata leaves rich in plant active substances (lactones, flavonoids, sterols) in goat diet increased the quantity of ruminal Ruminococcus albus, Ruminococcus flavefaciens, and Fibrobacter succinogenes, and then improved the nutrients digestibility [11]. The extracts of $P$. frutescens exhibit intense antimicrobial activities [12] and mitigate methane emissions from ruminants [13]. Many previous studies found that plant secondary metabolites, such as phenols, flavonoids, and triterpenes, have strong regulatory effects on ruminal microorganisms $[1,4,13,14]$.

To our knowledge, however, little research has been conducted to explore the effects of PFL on rumen fermentation and ruminal microbiota in dairy cows. In this study, we hypothesized that the PFL had effects on the rumen microbiota and rumen metabolism, possibly providing an efficient regulatory effect for the dairy cow feed industry. Therefore, this study was conducted to investigate the effects of dietary supplementation with PFL on rumen fermentation and ruminal bacterial community in dairy cows.

\section{Materials and Methods}

\subsection{Animals, Diets, and Experimental Design}

The use of the animals was approved by the Animal Care Committee of China Agricultural University (Beijing, China; approval no. AW26128102-1; approval date: 26 December 2018), and the experimental procedures used in this study were in accordance with the university's guidelines for animal research. The animals used in this study were from a commercial dairy farm with approximately 1500 lactating dairy cows (Shandong Yinxiangweiye dairy farm, Shandong, China). The animals used in this study were housed separately with other cows using a specific subfield. Thirty-six Holstein dairy cows (initial characteristics: milk yield $=42.1 \pm 8.70 \mathrm{~kg} / \mathrm{d}$; days in milk $=90.7 \pm 35.0 \mathrm{~d}$; parity $=3.0 \pm 1.35$; mean $\pm \mathrm{SD}$ ) were randomly divided into two treatment groups according to a randomized block design $(n=18)$. The treatment included a basal total mixed ration (TMR) diet without (CON group) or with dietary PFL at $300 \mathrm{~g} / \mathrm{d}$ per cow (PFL group). The ingredients and chemical composition of the basal TMR are shown in Supplemental Table S1. The adaptation period was one week, and the trial period was 8 weeks. At the end of the feeding experiment, fourteen cows (each group had seven cows) were selected for the collection of rumen fluid based on the milk yield (close to the average milk yield of the group).

\subsection{Sample Collection and Measurements}

The PFL was chemically characterized with the following procedure. The PFL sample ( $50 \mathrm{mg})$ was weighed into an EP tube, and $1000 \mu \mathrm{L}$ of extract solution (acetonitrile: methanol: water $=2: 2: 1$ ) with $1 \mu \mathrm{g} / \mathrm{mL}$ internal standard (2-chloro-L-phenylalanine, purity $\geq 98 \%$ ) was added. After $30 \mathrm{~s}$ of vortexing, the samples were homogenized at $35 \mathrm{~Hz}$ for $4 \mathrm{~min}$ and sonicated for $5 \mathrm{~min}$ on ice. The homogenization and sonication cycle was repeated 3 times. Then the samples were incubated for $1 \mathrm{~h}$ at $-20^{\circ} \mathrm{C}$ and centrifuged at $11,000 \mathrm{rpm}$ for $15 \mathrm{~min}$ at $4{ }^{\circ} \mathrm{C}$. The resulting supernatant was transferred to a fresh glass vial for LC/MS analysis. The detailed procedure of LC/MS analysis procedure was described in our previous study [1].

The individual milk yield was recorded every day with a milk-sampling device (Afkin Ranch Management Software, version 5.2, Kibbutz Afikim, Israel). During the experiment, a milk sample from one day ( $50 \mathrm{~mL}, 4: 3: 3$, composite) was collected every two weeks from three milking events and added to the composite milk sample, which was then stored at $4{ }^{\circ} \mathrm{C}$ for future milk protein, fat, lactose, total solid, and urea nitrogen analyses.

Ruminal fluid was collected $3 \mathrm{~h}$ after the morning feeding on the last day of this experiment using an oral stomach tube according to Shen et al. [15]. The initial $150 \mathrm{~mL}$ was discarded. The fluid $\mathrm{pH}$ was measured immediately. The samples were immediately flash-frozen in liquid nitrogen and stored at $-80{ }^{\circ} \mathrm{C}$ for DNA extraction, and another subsample was used to measure the volatile fatty acids 
(VFA) according to Hu et al. [16] and to determine the ammonia nitrogen according to Broderick and Kang [17].

\subsection{DNA Extraction and Sequencing}

Microbial DNA was extracted from rumen fluid $(1.5 \mathrm{~mL})$ samples using the E.Z.N.A. stool DNA kit (Omega Biotek, Norcross, GA, U.S.) according to the manufacturer's protocols. The quality of DNA was evaluated by $1 \%$ agarose gel electrophoresis and spectrophotometry. The final DNA concentration and purification were determined by a NanoDrop 2000 UV-VIS spectrophotometer (Thermo Scientific, Wilmington, DE, USA). The 16S rRNA V3-V4 region of the prokaryotic ribosomal RNA gene was amplified using the following primers: 338F: ACTCCTACGGGAGGCAGCAG and 806R: GGACTACHVGGGTWTCTAAT, where the barcode is an eight-base sequence that is unique to each sample [18]. The DNA template was uniformly diluted to $10 \mathrm{ng}$ for amplification. The PCR procedure was as follows: $95^{\circ} \mathrm{C}$ for $3 \mathrm{~min}$, followed by 27 cycles at $95^{\circ} \mathrm{C}$ for $10 \mathrm{~s}, 55^{\circ} \mathrm{C}$ for $30 \mathrm{~s}$, and $72{ }^{\circ} \mathrm{C}$ for $45 \mathrm{~s}$, and a final extension at $72{ }^{\circ} \mathrm{C}$ for $10 \mathrm{~min}$; PCRs were performed in triplicate in a $20 \mu \mathrm{L}$ mixture containing $4 \mu \mathrm{L}$ of $5 \times$ FastPfu Buffer, $2 \mu \mathrm{L}$ of $2.5 \mathrm{mM}$ dNTPs, $0.8 \mu \mathrm{L}$ of each primer $(5 \mu \mathrm{M}), 0.4 \mu \mathrm{L}$ of FastPfu Polymerase and $10 \mathrm{ng}$ of template DNA. Amplicons were extracted from $2 \%$ agarose gels and purified using the AxyPrep DNA Gel Extraction Kit (Axygen Biosciences, Union City, CA, USA) and quantified using QuantiFluor ${ }^{\mathrm{TM}}-\mathrm{ST}$ (Promega, Madison, WI, USA) following the manufacturer's protocol. Purified amplicons were pooled equimolarly and paired-end sequenced $(2 \times 300)$ on an Illumina MiSeq PE300 platform (Illumina, San Diego, CA, USA) following the standard protocols by Majorbio Bio-Pharm Technology Co. Ltd. (Shanghai, China). The raw sequences were deposited in the NCBI Sequence Read Archive (https://www.ncbi.nlm.nih.gov/sra/SRP222284) under the accession number SRP222284.

\subsection{Bioinformatic Analysis}

Raw fastq files were quality-filtered by Trimmomatic and merged by FLASH: the reads were truncated at any site receiving an average quality score $<20$ over a $50 \mathrm{bp}$ sliding window; sequences whose overlap was longer than $10 \mathrm{bp}$ were merged according to their overlap with no more than $2 \mathrm{bp}$ mismatch; sequences of each sample were separated according to barcodes (exact matches) and primers (allowing for two nucleotide mismatches). Reads containing ambiguous bases were removed. Operational taxonomic units (OTUs) were clustered with $\geq 97 \%$ similarity cutoff using UPARSE (version 7.1; http://drive5.com/uparse/) with a novel 'greedy' algorithm that performs chimera filtering and OTU clustering simultaneously. Venn analysis was performed to identify unique and common OTUs between the CON and PFL groups. The taxonomy of each 16S rRNA gene sequence was analyzed by RDP Classifier algorithm (http://rdp.cme.msu.edu/; Version 2.2) based on the SILVA database (https://www.arb-silva.de/). The confidence used for the classification is 0.7. Chao1, Simpson and all other alpha diversity indices were calculated in QIIME. The sequences were rarefied at the same value $(25,965)$ for each sample.

The OTU rarefaction curve and rank-abundance curves were plotted in QIIME. A Kruskal-Wallis $\mathrm{H}$ test and Tukey's HSD test were performed in R (Version 3.2.4, Auckland, New Zealand) to compare the alpha indices among groups. Multivariate statistical analyses, including a principal coordinate analysis (PCoA) were calculated and plotted in R (Version 3.2.4). The non-strict version of LEfSe was used to determine the bacteria most likely to explain the differences between PFL and CON by coupling a nonparametric factorial Kruskal-Wallis (KW) sum-rank test for statistical significance with additional tests assessing biological consistency and the relevance of effects. Bacteria with LDA scores greater than 3 were speculated to have a different abundance between the PFL and CON groups.

\subsection{Correlation and Statistical Analysis}

To explore the functional correlation between the rumen bacterial and rumen fermentation, and between rumen bacteria and milk synthesis, a Spearman's rank correlation matrix was generated by 
calculating the Spearman's correlation coefficient among the top 20 genera and candidate ruminal fermentation and milk performance variables in the R program, and only connections with a $p$-value of less than 0.05 and $r>0.54$ were retained. The rumen fermentation characteristics were analyzed with a randomized complete block design using PROC MIXED of SAS (version 9.2, SAS Institute Inc., Cary, NC, USA). The results were reported as least squares means that were calculated and separated using the PDIFF option in SAS. Significance was declared at a $p$-value $\leq 0.05$; trends were declared at $0.05<p$-value $\leq 0.10$.

\section{Results}

\subsection{Characteristics of PFL}

We found 122 bioactive compounds in PFL using UHPLC-QTOF-MS (Supplemental Table S2). The top 10 enriched bioactive compounds were clareolide, betaine, sucrose, scutellarin, apigenin, L-valine, caffeic acid, 2-pyrrolidinecarboxylic acid, L-phenylalanine, and L-tryptophan. Most of these compounds are flavonoids, alkaloid, triterpenoids, amino acids, etc.

\subsection{Milk Performance and Rumen Fermentation}

Feed intake was not affected by diet, but it was affected by week $(p<0.01$, Table 1$)$. Milk yield was higher in the PFL group than in the CON group $(p=0.04)$, and was greater in cows fed PFL than in cows fed CON after 3 weeks of feeding $(p<0.01)$. Compared to the control group, the yield of milk protein $(p=0.04)$ and lactose $(p=0.01)$ were increased in the PFL group. A higher level of feed efficiency (milk yield/dry matter intake) was detected in the PFL cows than in the control cows $(p<0.01)$. No interactive effects between sampling time and treatment were observed for the abovementioned variables $(p>0.05)$.

Rumen $\mathrm{pH}$ was higher in PFL cows than in CON cows (Table 2). The molar proportion of the valerate concentration had a tendency to be higher in the PFL group than in the CON group $(p=0.08)$.

Table 1. Effect of Perilla frutescens leaf (PFL) supplementation on feed intake and lactation performance in dairy cows.

\begin{tabular}{|c|c|c|c|c|c|c|}
\hline \multirow{2}{*}{ Item } & \multirow{2}{*}{$\mathrm{CON}$} & \multirow{2}{*}{ PFL } & \multirow{2}{*}{ SEM } & \multicolumn{3}{|c|}{$p$-Value } \\
\hline & & & & Treatment & Week & Treatment $\times$ Week \\
\hline Dry matter intake, $\mathrm{kg} / \mathrm{d}$ & 24.3 & 24.7 & 0.17 & 0.23 & $<0.01$ & 0.63 \\
\hline \multicolumn{7}{|c|}{ Milk yield, kg/d } \\
\hline Raw & 36.8 & 39.2 & 0.77 & 0.04 & $<0.01$ & 0.12 \\
\hline $\mathrm{ECM}^{\mathrm{a}}$ & 45.4 & 47.3 & 1.34 & 0.35 & 0.04 & 0.57 \\
\hline Protein & 1.28 & 1.36 & 0.026 & 0.04 & 0.10 & 0.73 \\
\hline Fat & 1.76 & 1.78 & 0.078 & 0.91 & $<0.01$ & 0.41 \\
\hline Lactose & 1.84 & 2.05 & 0.046 & 0.01 & 0.10 & 0.77 \\
\hline Total solids & 4.98 & 5.27 & 0.127 & 0.12 & 0.07 & 0.66 \\
\hline \multicolumn{7}{|c|}{ Milk content, g/100g } \\
\hline Protein & 3.47 & 3.39 & 0.037 & 0.19 & $<0.01$ & 0.58 \\
\hline Fat & 4.80 & 4.40 & 0.187 & 0.15 & $<0.01$ & 0.09 \\
\hline Lactose & 5.05 & 5.10 & 0.025 & 0.18 & 0.60 & 0.08 \\
\hline TS & 13.5 & 13.1 & 0.21 & 0.19 & $<0.01$ & 0.16 \\
\hline Milk urea nitrogen, mg/dL & 16.3 & 16.8 & 0.34 & 0.34 & $<0.01$ & 0.93 \\
\hline Fat: Protein & 1.38 & 1.30 & 0.051 & 0.30 & $<0.01$ & 0.16 \\
\hline Somatic cell count, $\times 10^{3}$ & 386.6 & 269.9 & 109.74 & 0.46 & 0.89 & 0.99 \\
\hline Feed efficiency ${ }^{b}$ & 1.51 & 1.63 & 0.011 & $<0.01$ & $<0.01$ & 0.48 \\
\hline
\end{tabular}

a $\overline{\mathrm{ECM}}=$ Energy-corrected milk. ECM $(\mathrm{kg})=0.3246 \times$ milk yield $(\mathrm{kg})+13.86 \times$ fat yield $(\mathrm{kg})+7.04 \times$ protein yield $(\mathrm{kg}) ;{ }^{\mathrm{b}}$ Feed efficiency $=$ milk yield/dry matter intake. 
Table 2. Comparison of rumen fermentation variables in dairy cows fed Perilla frutescens leaf (PFL) and control (CON) diets.

\begin{tabular}{|c|c|c|c|c|c|c|}
\hline \multirow{2}{*}{ Items } & \multicolumn{4}{|c|}{ Treatments $^{a}$} & \multirow{2}{*}{ SEM } & \multirow{2}{*}{$p$-Value } \\
\hline & $\mathrm{CON}$ & SD & PFL & SD & & \\
\hline $\mathrm{pH}$ & 6.20 & 0.23 & 6.47 & 0.22 & 0.075 & 0.04 \\
\hline Ammonia-nitrogen, $\mathrm{mg} / \mathrm{dL}$ & 9.97 & 4.99 & 8.66 & 2.20 & 1.825 & 0.63 \\
\hline \multicolumn{7}{|l|}{ Concentration, $\mathrm{mmol} / \mathrm{L}$} \\
\hline Total VFA ${ }^{a}$ & 67.8 & 13.1 & 71.5 & 15.5 & 6.33 & 0.70 \\
\hline Acetate & 42.6 & 9.56 & 42.8 & 9.34 & 4.12 & 0.96 \\
\hline Propionate & 15.7 & 2.91 & 18.2 & 3.78 & 1.52 & 0.30 \\
\hline Butyrate & 7.39 & 1.58 & 8.15 & 2.11 & 0.741 & 0.50 \\
\hline Valerate & 0.99 & 0.22 & 1.15 & 0.28 & 0.099 & 0.29 \\
\hline Isobutyrate & 0.41 & 0.09 & 0.43 & 0.12 & 0.052 & 0.79 \\
\hline Isovalerate & 0.7 & 0.22 & 0.69 & 0.25 & 0.12 & 0.94 \\
\hline \multicolumn{7}{|l|}{ Molar proportion, $\%$} \\
\hline Acetate & 62.6 & 3.92 & 59.9 & 1.35 & 1.1 & 0.14 \\
\hline Propionate & 23.4 & 3.50 & 25.6 & 1.85 & 1.13 & 0.23 \\
\hline Butyrate & 10.9 & 0.95 & 11.3 & 0.62 & 0.22 & 0.20 \\
\hline Valerate & 1.46 & 0.19 & 1.61 & 0.14 & 0.05 & 0.08 \\
\hline Isobutyrate & 0.6 & 0.07 & 0.6 & 0.14 & 0.05 & 0.98 \\
\hline Isovalerate & 1.03 & 0.20 & 0.96 & 0.31 & 0.129 & 0.73 \\
\hline Acetate: propionate & 2.74 & 0.55 & 2.35 & 0.21 & 0.162 & 0.14 \\
\hline
\end{tabular}

${ }^{\text {a }}$ VFA = volatile fatty acids; SD = standard deviations; SEM = standard error mean

\subsection{Change in Ruminal Bacterial Communities}

The number of sequences (total and average) before filtering is 797,635 and 56,974, respectively. The number of sequences (total and average) after filtering is 455,756 and 32,554, respectively. There were 1858 OTUs that were identified in PFL and CON cows, among which 1670 OTUs were found in both groups and accounted for $90.0 \%$ of the total OTUs, indicating the presence of an extensive common microbiome (Figure 1A). Compared with the CON, the PFL group had more OTUs $(p<0.01$; Figure 1A). The PCoA based on Bray-Curtis dissimilarity was applied to analyze the difference in bacterial composition between PFL and CON groups. The PCoA plots (Figure 1B) showed that the clouds derived from the PFL and CON data had a tendency to be separated from each other. A similar level of species richness existed between PFL and CON based on the Sobs, Shannon, Simpson, Ace, Chao, and coverage index analyses, which indicated that there was a similar tendency of diversity and uniformity between PFL and CON (Table 3).

In total, seven bacterial phyla were identified in the rumen samples that had relatively high abundances ( $>1 \%)$, including Bacteroidetes, Firmicutes, Proteobacteria, Patescibacteria, Spirochaetes, Tenericutes, and Actinobacteria (Figure 2A). There were 247 bacterial taxa identified at the genus level, and 27 genera were present with relatively high abundances $(>1 \%$, Figure $2 \mathrm{~B})$. For the genus level (Figure 2B), the percentage of unclassified sequences is $8.1 \%$ (20/247).

We identified 25 clades as PFL biomarkers, which distinguished the PFL group from the CON group (Figure 3). Twenty clades were more abundant in the PFL samples, including four genera belonging to the Clostridiales (Marvinbryantia, Acetitomaculum, Ruminococcus gauvreauii, Eubacterium coprostanoligenes), one genus belonging to the Veillonellaceae (Selenomonas_1), one genus belonging to the 
Bifidobacteriaceae (Pseudoscardovia), one genus belonging to Muribaculaceae (norank $f \_$Muribaculaceae), and Sharpea. In addition, at the order level, Clostridiales, Selenomonadales, and Bifidobacteriales were affected by PFL compared to the CON. At the class level, Clostridia, Negativicutes, and Actinobacteria were different between the PFL and CON. At the phylum level, Firmicutes and Actinobacteria were different between PFL and CON groups. Five clades were much less abundant in the PFL samples, including Treponema_2 belonging to Spirochaetaceae at the family level, Spirochaetales at the order level, Spirochaetia at the class level, and Spirochaetes at the phylum level.

Table 3. Alpha diversity indices of rumen bacteria in dairy cows fed Perilla frutescens leaf (PFL) and control (CON) diets.

\begin{tabular}{ccccc}
\hline \multirow{2}{*}{ Items } & \multicolumn{2}{c}{ Treatments } & \multirow{2}{*}{ SEM ${ }^{\mathbf{a}}$} & $p$-Value \\
\cline { 2 - 3 } & CON & PFL & & \\
\hline Sobs & 1147 & 1171 & 79.538 & 0.62 \\
Shannon & 5.12 & 5.58 & 0.429 & 0.13 \\
Simpson & 0.05 & 0.01 & 0.038 & 0.16 \\
Ace & 1379 & 1385 & 70.5 & 0.87 \\
Chao & 1394 & 1386 & 69.4 & 0.84 \\
Coverage & 0.99 & 0.99 & 0.001 & 0.31 \\
\hline \multicolumn{5}{c}{}
\end{tabular}

\subsection{Correlation Analysis}

It was found that the rumen fermentation indices were related to the ruminal bacteria community (Figure 4A). In detail, Christensenellaceae_R-7 $(r=0.58, p<0.05)$, Eubacterium coprostanoligenes $(r=0.62$, $p<0.05)$, and norank $f \_$Muribaculaceae $(r=0.75, p<0.01)$ were positively correlated with $\mathrm{pH}$ value. Prevotellaceae_UCG-003 $(\mathrm{r}=0.54, p<0.05)$ and Prevotella_1 $(r=0.56, p<0.05)$ were positively correlated with ammonia nitrogen. Prevotella_1 $(r=0.67, p<0.01)$ and unclassified_f_Prevotellaceae $(r=0.60, p<0.05)$ were positively correlated with acetate. Prevotella_1 $(\mathrm{r}=0.79, p<0.01)$ and unclassified_f_Prevotellaceae $(r=0.76, p<0.01)$ were positively correlated with butyrate. Prevotella_1 $(\mathrm{r}=0.68, p<0.01)$, Succiniclasticum $(\mathrm{r}=0.66, p<0.05)$, unclassified_f_Prevotellaceae $(r=0.79, p<0.01)$, and Ruminococcus_2 $(r=0.62, p<0.05)$ were positively correlated with valerate. Prevotella_1 $(r=0.65$, $p<0.05)$ and unclassified_f_Prevotellaceae $(r=0.56, p<0.05)$ were positively correlated with total volatile fatty acids. Rikenellaceae_RC9_gut $(r=0.59, p<0.05)$, Prevotellaceae_UCG-001 ( $\mathrm{r}=0.67, p<0.05)$, and Prevotellaceae_UCG-003 $(\mathrm{r}=0.76, p<0.05)$ were positively correlated, but Succinivibrionaceae_UCG-001 $(r=-0.57, p<0.05)$, Prevotella_7 $(r=-0.58, p<0.05)$, and Lachnospira $(\mathrm{r}=-0.66, p<0.05)$ were negatively correlated with acetate: propionate.

At the genus level, norank $\_\_$Muribaculaceae $(r=0.68, p<0.01)$ and Acetitomaculum $(r=0.59$, $p<0.05)$ were positively correlated with milk yield (Figure 4B). norank_f_Muribaculaceae $(r=0.63$, $p<0.05)$ was positively correlated but Treponema_2 $(r=-0.64, p<0.05)$ was negatively correlated with energy corrected milk. Eubacterium coprostanoligenes was negatively correlated with milk fat content $(\mathrm{r}=0.54, p<0.05)$. Prevotellaceae_UCG-003 $(r=-0.57, p<0.05)$ and norank_f_F082 $(r=-0.54, p<0.05)$ were negatively correlated with milk lactose content. Succinivibrionaceae_UCG-001 $(r=-0.55, p<0.05)$ was negatively correlated with milk urea nitrogen. 

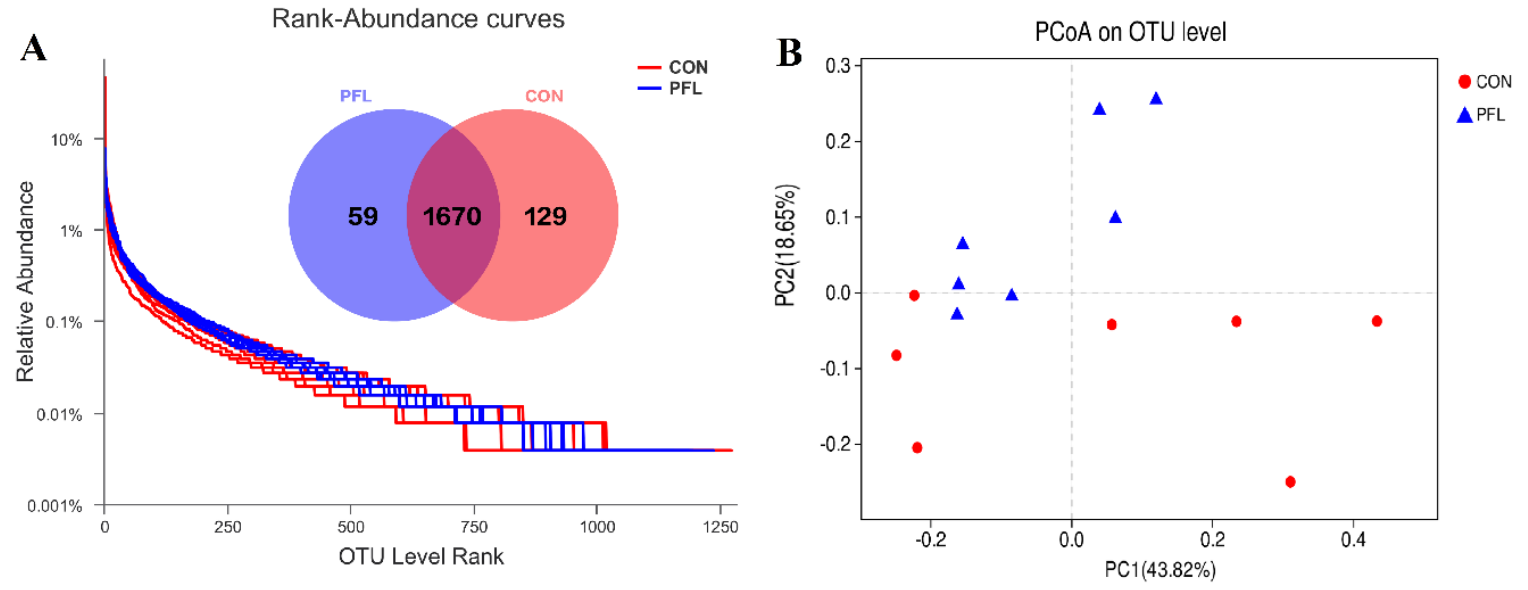

Figure 1. The rank-abundance curves derived from the OTU level. Venn diagram illustrating overlap of microbial operational taxonomic units (OTUs) at the 3\% dissimilarity level among treatments (A). Unweighted principal coordinate analysis (PCoA) analysis of taxonomical classifications of rumen bacterial communities in the cows fed P. frutescens leaf (PFL) and control (CON) diets (B).
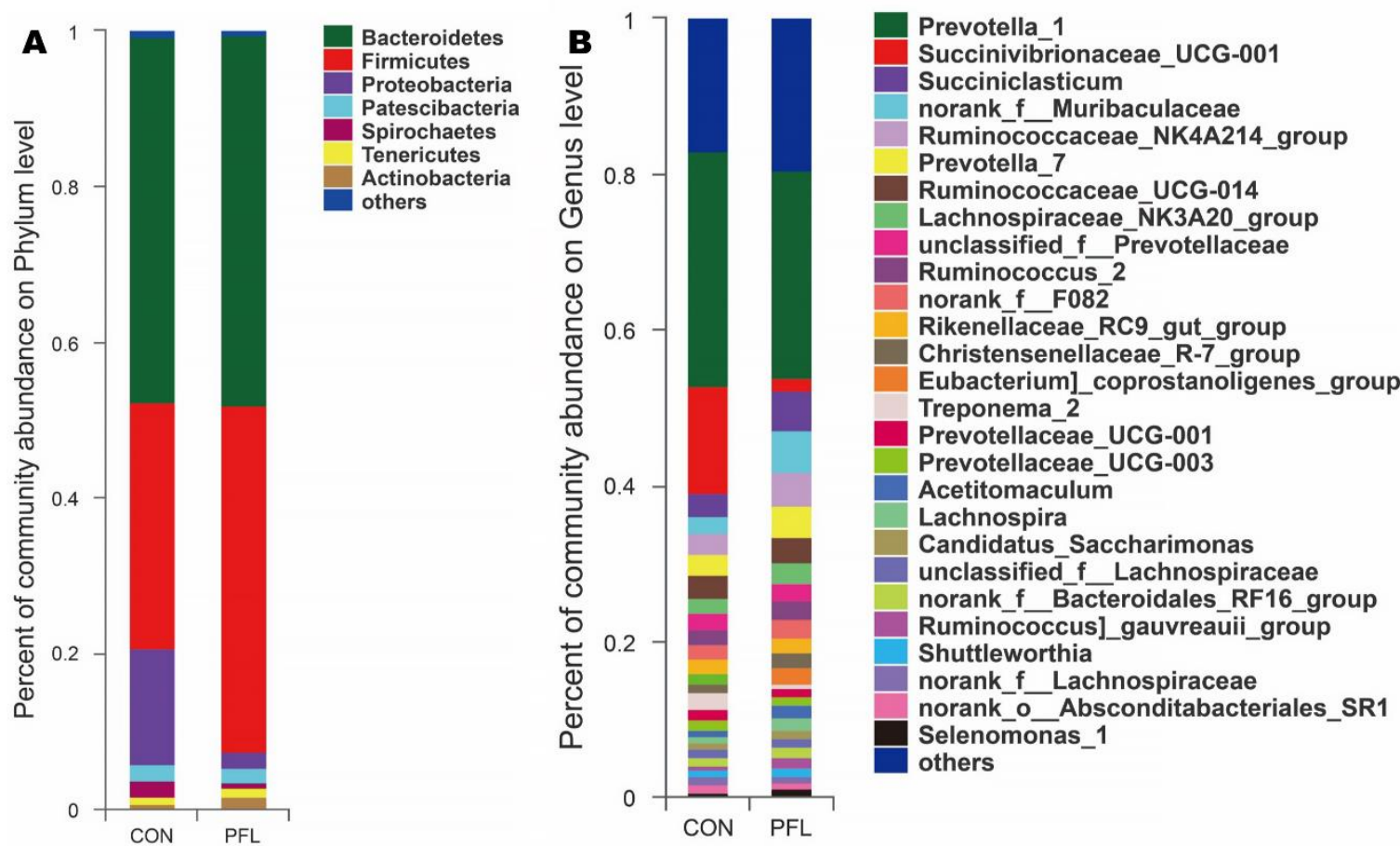

Figure 2. Distribution of bacterial taxa averaged under phyla (A) and genera (B) level across the dietary treatments (as a percentage of the total sequence). 


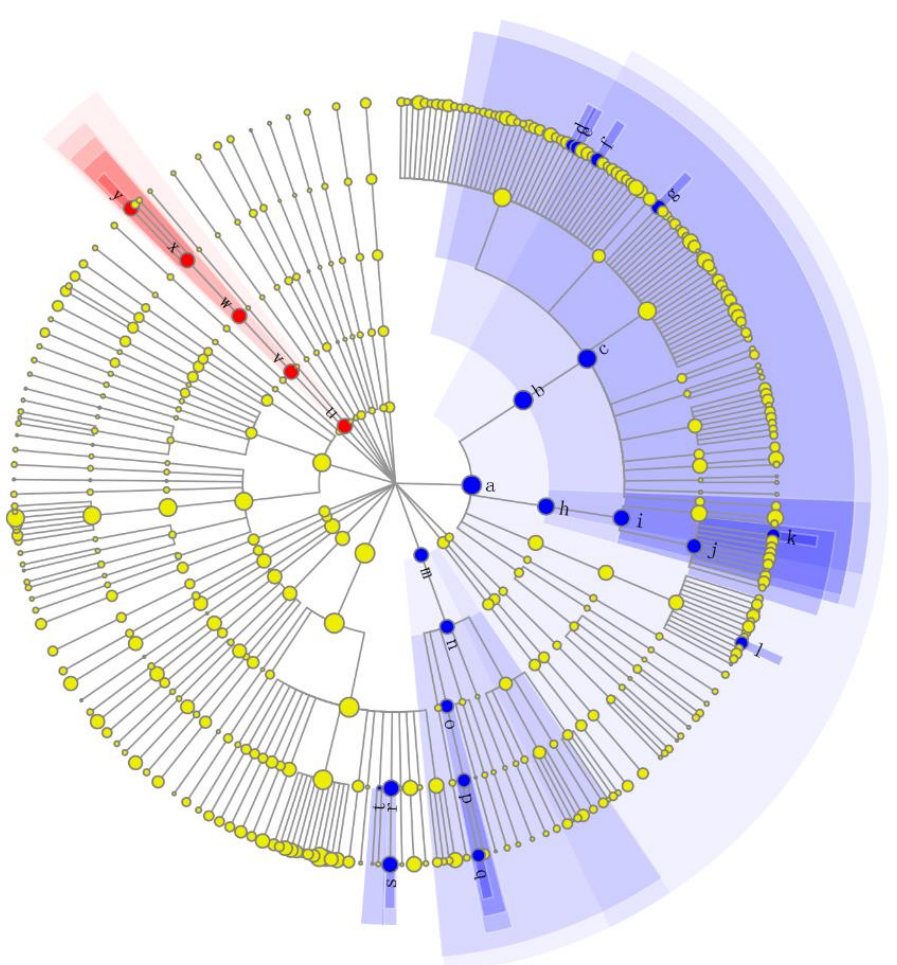

f : g_Ruminococcus_gauvreaui__group

m : p_Actinobacteria

Figure 3. The ruminal bacteria (highlighted by small circles and by shading) showing different abundance values between Perilla frutescens leaf (PFL) and control (CON) groups. There are six layers from the inside of this plot to the outside, corresponding to six levels of taxonomy (kingdom, phylum, class, order, family, and genus). Each node (small circle) represents a taxon. Blue and red nodes represent the bacterial community of PFL with the significant higher and lower abundance in PFL compared with that in the CON group, respectively. Yellow nodes indicate the bacteria that are not statistically and biologically differentially between the two groups. The diameter of each circle is proportional to the taxon's abundance. 

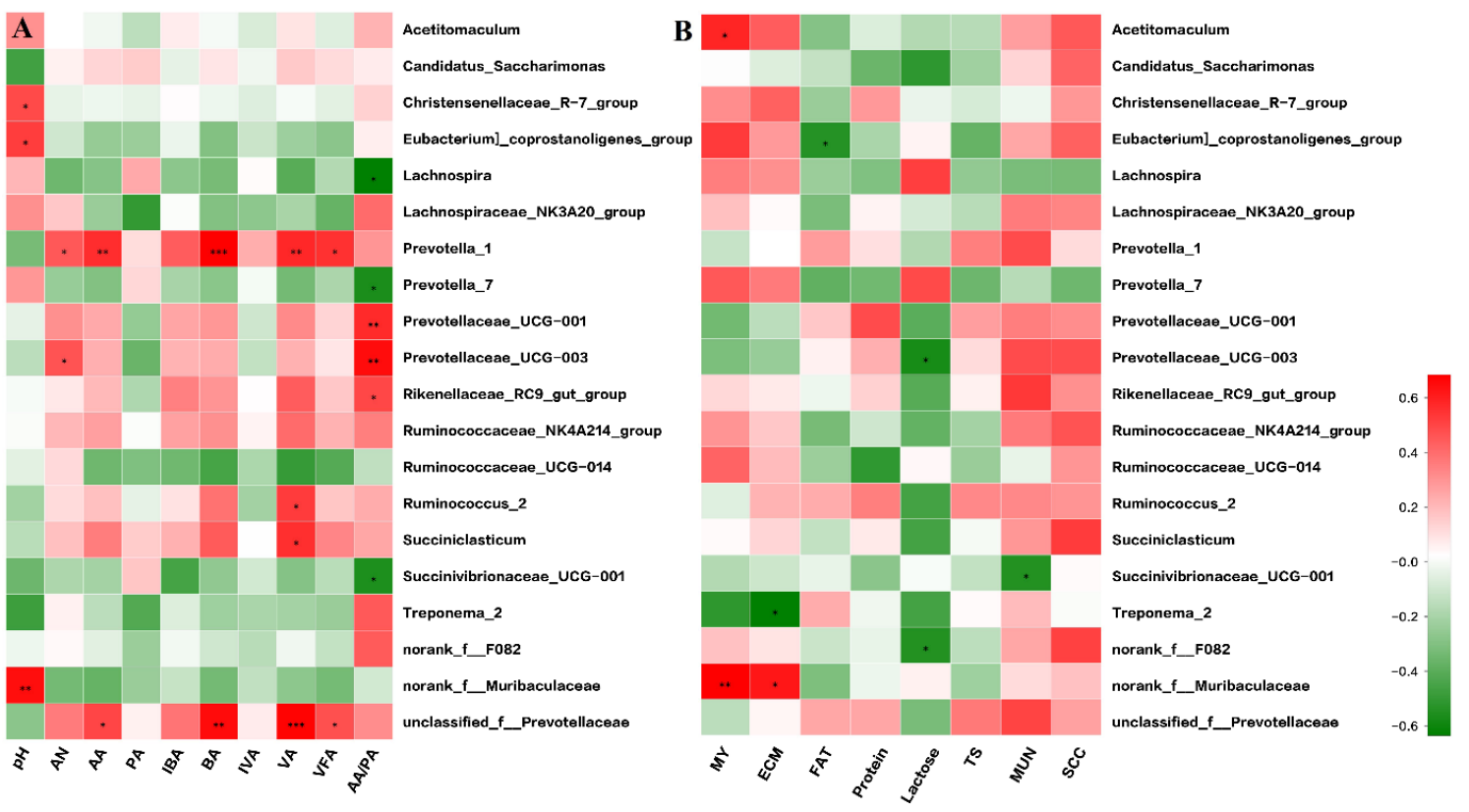

Figure 4. Correlation matrix between the top 20 ruminal bacterial genera and rumen fermentation parameters (A) and between the top 20 ruminal bacterial genera and milk performance variables (B). Positive correlations are shown in red and negative correlations are shown in green. The color intensity is proportional to the correlation values [r] within a correlation group. ${ }^{*} p<0.05 ;{ }^{* *} p<0.01$. $\mathrm{AN}=$ ammonia nitrogen, $\mathrm{AA}=$ acetate, $\mathrm{PA}=$ propionate, $\mathrm{IBA}=$ isobutyrate, $\mathrm{BA}=$ butyrate, $\mathrm{VA}=$ valerate, IVA $=$ isovalerate, VFA $=$ total volatile fatty acids, AA_PA $=$ the ratio of acetate to propionate, $\mathrm{MY}=$ milk yield, ECM = energy corrected milk, TS = total solids, MUN = milk urea nitrogen, SCC = somatic cell count.

\section{Discussion}

The current study characterized the changes in milk performance and rumen bacterial composition and the linkage between these factors after cows were fed PFL. The PFL has been reported to mainly contain hydrophilic (phenolic compounds, flavonoids, and triterpenes) and hydrophobic compounds (volatile compounds, fatty acids, policosanols, tocopherols, and phytosterols) [4], which was similar to the results of our study. In our previous study, when dairy cows were fed triterpene saponins at a moderate dose, milk synthesis increased and immune function improved [19]. In addition, the ethanol extract of $P$. frutescens seeds used at a relatively low concentration decreased methane production without adversely affecting rumen fermentation [13]. Considering the characteristics of the herb used, we estimated that the increased milk yield and feed efficiency might be due to the beneficial role of the bioactive compounds of PFL in modifying rumen fermentation by inhibiting energy transfer to the methane production pathway.

Furthermore, we identified specific rumen bacteria that may contribute to rumen fermentation and milk performance. Our results revealed that the addition of PFL had a positive effect on Firmicutes, Actinobacteria, and Spirochaetes, but had no effects on Bacteroidetes and Proteobacteria phyla compared with CON. In addition, we found that the relative abundance of Marvinbryantia, Acetitomaculum, Ruminococcus gauvreauii, Eubacterium coprostanoligenes, Selenomonas_1, Pseudoscardovia, norank $f \_$_Muribaculaceae, and Sharpea was higher, but that of Treponema_2 was lower in the PFL than in the CON. In addition, Acetitomaculum, Eubacterium coprostanoligenes, norank $f$ _Muribaculaceae, and Treponema_2 ranked among the top 20 genera and were highly correlated with milk performance. Thus, these four bacterial taxa may be associated with PFL.

The three dominant ruminal bacterial phyla in the current study were Bacteroidetes, Proteobacteria, and Firmicutes, which was similar to the findings of previous studies [20,21]. The phylum Firmicutes was increased after cows were fed PFL in the present study. There were close associations between 
Firmicutes and the degradation of structural polysaccharides [22]. We also found that the proportion of valerate in cows fed PFL tended to be greater than that in CON cows. Valerate can be produced by ruminal bacterial species of Clostridium, and the abundance of Clostridium was negatively associated with methane yield [23]. The class Clostridia and order Clostridiales belonging to Firmicutes were increased after cows were fed PFL. In addition, Marvinbryantia, Acetitomaculum, Ruminococcus gauvreauii, Eubacterium coprostanoligenes, Selenomonas_1, and Sharpea, belonging to Firmicutes, were increased after cows were fed PFL. Thus, we propose that the PFL might increase the ruminal degradation of structural polysaccharides accompanied by potential inhibition of methane production. However, the assumption should be confirmed by further research.

We found that the relative abundance of norank $f \_$_Muribaculaceae was highly positively correlated with the ruminal $\mathrm{pH}$ value, which was significantly greater in cows fed PFL than in CON cows. The abundance of Muribaculaceae was an important predictor of short chain fatty acid concentrations in the guts of mice [24]. Metagenomics results suggested that populations of Muribaculaceae are equipped with fermentation pathways to produce succinate, acetate, and propionate [25] through the degradation of particular types of polysaccharides, including plant glycans, host glycans, and $\alpha$-glucans $[25,26]$. Thus, Muribaculaceae has the ability to undergo acetogenesis, similar to Acetitomaculum, which belongs to the group of acetogenic bacteria, which are a diverse group of bacteria whose main unifying characteristics is the ability to synthesize acetate from $\mathrm{H}_{2}+\mathrm{CO}_{2}$ [27]. As the abundance of Acetitomaculum increased in the current study, the competitive advantage of acetogenesis may have increased compared with that of methanogenesis, and this may reduce the energy lost as methane as well as the eructation of ruminal methane as a greenhouse gas [28]. In a previous study, the abundances of Acetitomaculum increased with increasing dietary energy [29]. Acetitomaculum mainly exists in ruminants fed a high-concentrate diet and can utilize monosaccharides to produce acetate [30]. In addition, the current study also found that norank $f$ __Muribaculaceae and Acetitomaculum were highly positively correlated with milk yield, indicating their potential role as bacterial biomarkers for milk synthesis. We also proposed that the cows fed PFL showed an increased energy allocation for milk synthesis via the increase in the abundance of ruminal Muribaculaceae and Acetitomaculum.

We found that the relative abundance of Eubacterium coprostanoligenes was increased by feeding cows PFL, which was also positively correlated with ruminal $\mathrm{pH}$ value, but negatively correlated with milk fat content. Our result was similar to a previous study that found that the abundances of Eubacterium coprostanoligenes were significantly higher in the high-yield dairy cow group than in the low yield group, and the high-yield had better ruminal fermentation patterns than the low yield cows [31]. In addition, our results revealed that the addition of PFL had a positive effect on $\mathrm{pH}$ value, which also showed that the increased $\mathrm{pH}$ value was associated with Eubacterium coprostanoligenes. To date, very few studies have focused the function of Eubacterium coprostanoligenes. Thus, the association between Eubacterium coprostanoligenes and milk fat and its relationship with $\mathrm{pH}$ requires further study. Quercetin did not prevent the growth of Ruminococcus gauvreauii [32]. In contrast, its aglycone quercetin exerted a strong inhibitory effect on Ruminococcus gauvreauii [33]. Naringenin strongly inhibited the growth of Ruminococcus gauvreauii, in the human gut [34]. In the present study, the PFL was found to be enriched with flavonoids, alkaloids, triterpenoids, etc., which was similar to the results of previous studies [3,4]. However, we found that the feeding of PFL to cows resulted in an increased abundance of Ruminococcus gauvreauii. To date, few studies have revealed the function of Ruminococcus gauvreauii [34]. We estimated that the increased abundance of Ruminococcus gauvreauii was due to the specific compounds in PFL, but this needs to be explored further.

In our study, an increased abundance of Sharpea in cows fed PFL was found. Bacteria belonging to Sharpea have been reported to utilize a wide variety of carbohydrates to produce lactate [35]. It was reported that the ruminal Sharpea of sheep produced low levels of methane [23]. A recent study found that the abundance of the genus Sharpea was much higher in the high milk yield and high milk protein content cows than in the low milk yield and low milk protein content cows [21], which was confirmed by the current study that the PFL cows with higher milk yield had greater abundance of ruminal Sharpea. 
In addition, Selenomonas that is also a lactate-utilizing bacteria [36] had greater abundance in PFL cows compared to CON cows. It was reported that Selenomonas_1 abundance was positively correlated with C18:1n9t concentration, and Selenomonas_1 participated in hydrogenation of long-chain fatty acids [29]. Thus, the increased abundance of Selenomonas_1 as a result of feeding cows PFL might have had positive effects on milk fat synthesis in increasing the milk unsaturated fatty acid synthesis, but this speculation needs further validation via the characterization of the milk fatty acid composition and its relationship with ruminal bacteria. In addition, our finding of the increased abundance of Selenomonas_1 in cows fed PFL was similar to a previous study that found that the extract of Perilla frutescens seeds effectively increased the abundance of Selenomonas [13]. We estimated that the cows fed PFL experienced a fermentation shift via the increased abundance of ruminal Sharpea and Selenomonas_1, thus contributing to higher milk yield [21]. However, this is just a speculation which should be confirmed by further studies for the functions of these bacteria in relating to milk performance.

A negative correlation was found between energy corrected milk and Treponema_2, and the relative abundance of Treponema_2 was lower in PFL than in CON. It was reported that Treponema are cellulose-degrading microbes [37]. Treponema are a commonly detected bacterial group in the rumen that are involved in the degradation of soluble fibers [38]. However, a high-concentrate diet significantly decreased the abundance of Treponema compared with a high fiber diet [39]. The growth of the Treponema genus was remarkably supported by the inclusion of pectin [40]. However, to our knowledge, no previous study has found inhibitory effects on Treponema using PFL or its extract. Thus, the underlying mechanism of PFL in inhibiting Treponema should be revealed. It was only reported that several plant extracts had the inhibition roles of peptidase and giycosidase activities of Treponema denticola [41]. Based on the previous studies, we hypothesized that the inhibition of Treponema might be due to the specific bioactive compounds in PFL.

In addition to the different bacterial taxa between PFL and CON, Succinivibrionaceae_UCG-001 and Prevotella_1 were correlated with milk synthesis or rumen fermentation characteristics. Succinivibrionaceae_UCG-001 comprised the core microbiome in young cattle [1], which was similar to the current study in adult cows. Members of the Succinivibrionaceae have been previously proposed to be responsible for the lower methane emissions in wallaby microbiota [42]. It was proposed that Succinivibrionaceae produce succinate which is converted to propionate by other members of the microbiota; thus, less hydrogen might be available for methanogens [43]. Thus, Succinivibrionaceae_UCG-001 was negatively correlated with the ratio of acetate to propionate in the current study, which might have been due to the increased production of propionate in the rumen. In addition, its highly negative correlation with milk urea nitrogen might be attributed to the improved nitrogen utilization efficiency because of the energy saved by the decrease in methane production [44]. Our results showed that Prevotella 1 had positive effects on VFA concentrations (acetate, propionate, and valerate), which was similar to a previous study that found a significant positive correlation between Prevotella and VFA [21]. Prevotella is a genus consisting of proteolytic, amylolytic, and hemicellulolytic bacteria dominating the rumen of adult dairy cows and producing succinate and acetate [45]. Therefore, the correlation analysis from the current study confirmed the function of Succinivibrionaceae_UCG-001 and Prevotella.

\section{Conclusions}

In this study, PFL was found to be enriched with flavonoids, alkaloids, triterpenoids, amino acids, etc., and induced compositional shifts in the ruminal microbiota, as confirmed for the first time by high-throughput sequencing, indicating that PFL is a potential functional feed source or additive that can be used to regulate rumen fermentation. We identified several key differential bacteria that responded to PFL addition, such as norank_f_Muribaculaceae and Acetitomaculum, which were also correlated with milk yield. In the future, it may be possible to reveal the functional microbiome associated with PFL or its key secondary metabolites through metagenomics or meta-transcriptomics. 
Supplementary Materials: The following are available online at http://www.mdpi.com/2076-2607/7/11/562/s1.

Author Contributions: B.W. designed the study. Z.S., Z.Y., and B.W. performed the sequencing analyses. Z.S. and B.W. analyzed the data. Z.S. and B.W. wrote the manuscript. All authors read the final version of manuscript and approved it.

Funding: This work was supported in part by the National Natural Science Foundation of China (31902181).

Acknowledgments: We would like to thank the personnel of Shandong Yinxiang Weiye Dairy Farm (Heze, China) for their assistance in feeding and care of the animals.

Conflicts of Interest: The authors declare no conflict of interest.

\section{References}

1. Wang, B.; Ma, M.; Diao, Q.; Tu, Y. Saponin-Induced Shifts in the Rumen Microbiome and Metabolome of Young Cattle. Front. Microbiol. 2019, 10, 356. [CrossRef] [PubMed]

2. Kamra, D.; Patra, A.; Chatterjee, P.; Kumar, R.; Agarwal, N.; Chaudhary, L. Effect of plant extracts on methanogenesis and microbial profile of the rumen of buffalo: A brief overview. Aust. J. Exp. Agr. 2008, 48, 175-178. [CrossRef]

3. Jun, H.-I.; Kim, B.-T.; Song, G.-S.; Kim, Y.-S. Structural characterization of phenolic antioxidants from purple perilla (Perilla frutescens var. acuta) leaves. Food Chem. 2014, 148, 367-372. [CrossRef]

4. Yu, H.; Qiu, J.-F.; Ma, L.-J.; Hu, Y.-J.; Li, P.; Wan, J.-B. Phytochemical and phytopharmacological review of Perilla frutescens L. (Labiatae), a traditional edible-medicinal herb in China. Food Chem. Toxicol. 2017, 108, 375-391. [CrossRef] [PubMed]

5. Mertenat, D.; dal Cero, M.; Vogl, C.R.; Ivemeyer, S.; Meier, B.; Maeschli, A.; Hamburger, M.; Walkenhorst, M. Ethnoveterinary knowledge of farmers in bilingual regions of Switzerland-is there potential to extend veterinary options to reduce antimicrobial use? J. Ethnopharmacol. 2019, 246, 112184. [CrossRef] [PubMed]

6. Hristov, A.N.; Oh, J.; Firkins, J.; Dijkstra, J.; Kebreab, E.; Waghorn, G.; Makkar, H.; Adesogan, A.; Yang, W.; Lee, C. Special topics-Mitigation of methane and nitrous oxide emissions from animal operations: I. A review of enteric methane mitigation options. J. Anim. Sci. 2013, 91, 5045-5069. [CrossRef]

7. Jami, E.; White, B.A.; Mizrahi, I. Potential role of the bovine rumen microbiome in modulating milk composition and feed efficiency. PLoS ONE 2014, 9, e85423. [CrossRef]

8. Huws, S.A.; Creevey, C.J.; Oyama, L.B.; Mizrahi, I.; Denman, S.E.; Popova, M.; Muñoz-Tamayo, R.; Forano, E.; Waters, S.M.; Hess, M. Addressing global ruminant agricultural challenges through understanding the rumen microbiome: Past, present, and future. Front. Microbiol. 2018, 9, 2161. [CrossRef]

9. Tan, J.; McKenzie, C.; Potamitis, M.; Thorburn, A.N.; Mackay, C.R.; Macia, L. The role of short-chain fatty acids in health and disease. Adv. Immunol. 2014, 121, 91-119.

10. Shen, H.; Lu, Z.; Xu, Z.; Chen, Z.; Shen, Z. Associations among dietary non-fiber carbohydrate, ruminal microbiota and epithelium G-protein-coupled receptor, and histone deacetylase regulations in goats. Microbiome 2017, 5, 123. [CrossRef]

11. Yusuf, A.L.; Adeyemi, K.D.; Samsudin, A.A.; Goh, Y.M.; Alimon, A.R.; Sazili, A.Q. Effects of dietary supplementation of leaves and whole plant of Andrographis paniculata on rumen fermentation, fatty acid composition and microbiota in goats. BMC Vet. Res. 2017, 13, 349. [CrossRef] [PubMed]

12. Seow, Y.X.; Yeo, C.R.; Chung, H.L.; Yuk, H.G. Plant essential oils as active antimicrobial agents. Crit. Rev. Food Sci. Nutr. 2014, 54, 625-644. [CrossRef] [PubMed]

13. Wang, J.; Liu, M.; Wu, Y.; Wang, L.; Liu, J.; Jiang, L.; Yu, Z. Medicinal herbs as a potential strategy to decrease methane production by rumen microbiota: A systematic evaluation with a focus on Perilla frutescens seed extract. Appl. Microbiol. Biot. 2016, 100, 9757-9771. [CrossRef] [PubMed]

14. Vasta, V.; Daghio, M.; Cappucci, A.; Buccioni, A.; Serra, A.; Viti, C.; Mele, M. Invited review: Plant polyphenols and rumen microbiota responsible for fatty acid biohydrogenation, fiber digestion, and methane emission: Experimental evidence and methodological approaches. J. Dairy Sci. 2019, 102, 3781-3804. [CrossRef]

15. Shen, J.; Chai, Z.; Song, L.; Liu, J.; Wu, Y. Insertion depth of oral stomach tubes may affect the fermentation parameters of ruminal fluid collected in dairy cows. J. Dairy Sci. 2012, 95, 5978-5984. [CrossRef]

16. Hu, W.-L.; Liu, J.-X.; Ye, J.-A.; Wu, Y.-M.; Guo, Y.-Q. Effect of tea saponin on rumen fermentation in vitro. Anim. Feed Sci. Technol. 2005, 120, 333-339. [CrossRef] 
17. Broderick, G.; Kang, J. Automated simultaneous determination of ammonia and total amino acids in ruminal fluid and in vitro media1. J. Dairy Sci. 1980, 63, 64-75. [CrossRef]

18. Xu, N.; Tan, G.C.; Wang, H.Y.; Gai, X.P. Effect of biochar additions to soil on nitrogen leaching, microbial biomass and bacterial community structure. Eur. J. Soil Biol. 2016, 74, 1-8. [CrossRef]

19. Wang, B.; Tu, Y.; Zhao, S.; Hao, Y.; Liu, J.; Liu, F.; Xiong, B.; Jiang, L. Effect of tea saponins on milk performance, milk fatty acids, and immune function in dairy cow. J. Dairy Sci. 2017, 100, 8043-8052. [CrossRef]

20. Klevenhusen, F.; Petri, R.M.; Kleefisch, M.-T.; Khiaosa-ard, R.; Metzler-Zebeli, B.U.; Zebeli, Q. Changes in fibre-adherent and fluid-associated microbial communities and fermentation profiles in the rumen of cattle fed diets differing in hay quality and concentrate amount. FEMS Microbiol. Ecol. 2017, 93. [CrossRef]

21. Xue, M.; Sun, H.; Wu, X.; Guan, L.; Liu, J. Assessment of rumen bacteria in dairy cows with varied milk protein yield. J. Dairy Sci. 2019, 102, 5031-5041. [CrossRef] [PubMed]

22. Zened, A.; Combes, S.; Cauquil, L.; Mariette, J.; Klopp, C.; Bouchez, O.; Troegeler-Meynadier, A.; Enjalbert, F. Microbial ecology of the rumen evaluated by 454 GS FLX pyrosequencing is affected by starch and oil supplementation of diets. FEMS Microbiol. Ecol. 2013, 83, 504-514. [CrossRef] [PubMed]

23. Kamke, J.; Kittelmann, S.; Soni, P.; Li, Y.; Tavendale, M.; Ganesh, S.; Janssen, P.H.; Shi, W.; Froula, J.; Rubin, E.M. Rumen metagenome and metatranscriptome analyses of low methane yield sheep reveals a Sharpea-enriched microbiome characterised by lactic acid formation and utilisation. Microbiome 2016, 4, 56. [CrossRef] [PubMed]

24. Smith, B.J.; Miller, R.A.; Ericsson, A.C.; Harrison, D.C.; Strong, R.; Schmidt, T.M. Changes in the gut microbiome and fermentation products concurrent with enhanced longevity in acarbose-treated mice. BMC Microbiol. 2019, 19, 130. [CrossRef] [PubMed]

25. Ormerod, K.L.; Wood, D.L.; Lachner, N.; Gellatly, S.L.; Daly, J.N.; Parsons, J.D.; Dal'Molin, C.G.; Palfreyman, R.W.; Nielsen, L.K.; Cooper, M.A. Genomic characterization of the uncultured Bacteroidales family S24-7 inhabiting the guts of homeothermic animals. Microbiome 2016, 4, 36. [CrossRef]

26. Lagkouvardos, I.; Lesker, T.R.; Hitch, T.C.; Gálvez, E.J.; Smit, N.; Neuhaus, K.; Wang, J.; Baines, J.F.; Abt, B.; Stecher, B. Sequence and cultivation study of Muribaculaceae reveals novel species, host preference, and functional potential of this yet undescribed family. Microbiome 2019, 7, 28. [CrossRef]

27. Ragsdale, S.W. Enzymology of the Wood-Ljungdahl pathway of acetogenesis. Ann. N. Y. Acad. Sci. 2008, 1125, 129. [CrossRef]

28. Martin, C.; Morgavi, D.; Doreau, M. Methane mitigation in ruminants: From microbe to the farm scale. Animal 2010, 4, 351-365. [CrossRef]

29. Wang, H.; He, Y.; Li, H.; Wu, F.; Qiu, Q.; Niu, W.; Gao, Z.; Su, H.; Cao, B. Rumen fermentation, intramuscular fat fatty acid profiles and related rumen bacterial populations of Holstein bulls fed diets with different energy levels. Appl. Microbiol. Biot. 2019, 1-12. [CrossRef]

30. Hua, C.; Tian, J.; Tian, P.; Cong, R.; Luo, Y.; Geng, Y.; Tao, S.; Ni, Y.; Zhao, R. Feeding a high concentration diet induces unhealthy alterations in the composition and metabolism of ruminal microbiota and host response in a goat model. Front. Microbiol. 2017, 8, 138. [CrossRef]

31. Tong, J.; Zhang, H.; Yang, D.; Zhang, Y.; Xiong, B.; Jiang, L. Illumina sequencing analysis of the ruminal microbiota in high-yield and low-yield lactating dairy cows. PLOS ONE 2018, 13, e0198225. [CrossRef] [PubMed]

32. Firrman, J.; Liu, L.; Zhang, L.; Argoty, G.A.; Wang, M.; Tomasula, P.; Kobori, M.; Pontious, S.; Xiao, W. The effect of quercetin on genetic expression of the commensal gut microbes Bifidobacterium catenulatum, Enterococcus caccae and Ruminococcus gauvreauii. Anaerobe 2016, 42, 130-141. [CrossRef] [PubMed]

33. Duda-Chodak, A. The inhibitory effect of polyphenols on human gut microbiota. J. Physiol. Pharmacol. 2012, 63, 497-503. [PubMed]

34. Watanabe, M.; Kaku, N.; Ueki, K.; Ueki, A. Falcatimonas natans gen. nov., sp. nov., a strictly anaerobic, amino-acid-decomposing bacterium isolated from a methanogenic reactor of cattle waste. Int. J. Syst. Evol. Micr. 2016, 66, 4639-4644. [CrossRef]

35. Kumar, S.; Treloar, B.P.; Teh, K.H.; McKenzie, C.M.; Henderson, G.; Attwood, G.T.; Waters, S.M.; Patchett, M.L.; Janssen, P.H. Sharpea and Kandleria are lactic acid producing rumen bacteria that do not change their fermentation products when co-cultured with a methanogen. Anaerobe 2018, 54, 31-38. [CrossRef]

36. Gutierrez, J. Numbers and characteristics of lactate utilizing organisms in the rumen of cattle. J. Bacterial. $1953,66,123$. 
37. Stanton, T.; Canale-Parola, E. Treponema bryantii sp. nov., a rumen spirochete that interacts with cellulolytic bacteria. Arch. Microbiol. 1980, 127, 145-156. [CrossRef]

38. Bekele, A.Z.; Koike, S.; Kobayashi, Y. Phylogenetic diversity and dietary association of rumen Treponema revealed using group-specific $16 S$ rRNA gene-based analysis. FEMS Microbiol. Lett. 2011, 316, 51-60. [CrossRef]

39. Luo, D.; Gao, Y.; Lu, Y.; Qu, M.; Xiong, X.; Xu, L.; Zhao, X.; Pan, K.; Ouyang, K. Niacin alters the ruminal microbial composition of cattle under high-concentrate condition. Anim. Nutr. 2017, 3, 180-185. [CrossRef]

40. Liu, J.; Pu, Y.-Y.; Xie, Q.; Wang, J.-K.; Liu, J.-X. Pectin induces an in vitro rumen microbial population shift attributed to the pectinolytic Treponema group. Curr. Microbiol. 2015, 70, 67-74. [CrossRef]

41. Homer, K.A.; Manji, F.; Beighton, D. Inhibition of peptidase and glycosidase activities of Porphyromonas gingivalis, Bacteroides intermedius and Treponema denticola by plant extracts. J. Clin. Periodontol. 1992, 19, 305-310. [CrossRef] [PubMed]

42. Pope, P.; Smith, W.; Denman, S.; Tringe, S.; Barry, K.; Hugenholtz, P.; McSweeney, C.; McHardy, A.; Morrison, M. Isolation of Succinivibrionaceae implicated in low methane emissions from Tammar wallabies. Science 2011, 333, 646-648. [CrossRef] [PubMed]

43. Santos, E.d.O.; Thompson, F. The family succinivibrionaceae. Prokaryotes: Gammaproteobacteria 2014, 639-648. [CrossRef]

44. Ramayo-Caldas, Y.; Zingaretti, L.; Popova, M.; Estellé, J.; Bernard, A.; Pons, N.; Bellot, P.; Mach, N.; Rau, A.; Roume, H. Identification of rumen microbial biomarkers linked to methane emission in Holstein dairy cows. J. Anim. Breed. Genet. 2019. [CrossRef]

45. Stevenson, D.M.; Weimer, P.J. Dominance of Prevotella and low abundance of classical ruminal bacterial species in the bovine rumen revealed by relative quantification real-time PCR. Appl. Microbiol. Biot. 2007, 75, 165-174. [CrossRef]

(C) 2019 by the authors. Licensee MDPI, Basel, Switzerland. This article is an open access article distributed under the terms and conditions of the Creative Commons Attribution (CC BY) license (http://creativecommons.org/licenses/by/4.0/). 